PROCEEDINGS OF THE AMERICAN MATHEMATICAL SOCIETY

Volume 124, Number 7, July 1996

\title{
HERMITE MULTIPLIERS AND PSEUDO-MULTIPLIERS
}

\author{
JAY EPPERSON
}

(Communicated by J. Marshall Ash)

\begin{abstract}
We prove a multiplier theorem for the Hermite-Triebel-Lizorkin spaces introduced by Epperson in [Studia Math. 114 (1995), 87-103]. This extends Thangavelu's theorem [Revist. Mat. Ibero 3 (1987), 1-24; Math. Notes, vol. 42, 1993] on Hermite multipliers for $L^{p}$ spaces. We also prove an $L^{p}$ boundedness result for a class of Hermite pseudo-multipliers.
\end{abstract}

\section{INTRODUCTION AND MAIN RESULTS}

We begin with a review of some of the notation and results from [1]. Consult [6] for background information on Hermite expansions. Let $h_{k}(x)$ denote the $k^{\text {th }} L^{2}(\mathbf{R})$-normalized Hermite function, $k \in \mathbf{N}_{0}=\{0,1,2, \ldots\}$. Recall that the collection $\left\{h_{k}\right\}_{k=0}^{\infty}$ is a complete orthonormal basis for $L^{2}$, and that $h_{k}(x)$ is an eigenfunction of the Hermite operator $H=-\frac{d^{2}}{d x^{2}}+x^{2}$ with corresponding eigenvalue $2 k+1$. If $m: \mathbf{R}_{+} \rightarrow \mathbf{C}$ is a bounded function, then we let $m(H)$ denote the bounded linear operator on $L^{2}$ defined by $m(H) h_{k}=m(2 k+1) h_{k}$.

Now suppose $\varphi: \mathbf{R} \rightarrow \mathbf{C}$ is $C^{\infty}$ and satisfies

$$
\begin{aligned}
& \text { (i) } \operatorname{supp} \varphi \subset\left[\frac{1}{2}, 2\right], \\
& \text { (ii) }|\varphi(x)| \geq c>0 \text { if } x \in\left[\frac{3}{4}, \frac{7}{4}\right] .
\end{aligned}
$$

For each $\mu \in \mathbf{N}_{0}$ define the operator $Q_{\mu}=\varphi\left(2^{-\mu} H\right)$. Let $L_{f}^{2}$ denote the space of finite linear combinations of Hermite functions. For $g \in L_{f}^{2}$ define the HermiteTriebel-Lizorkin norm

$$
\|g\|_{H_{p}^{\alpha q}}=\left\|\left(\sum_{\mu=0}^{\infty}\left(2^{\mu \alpha}\left|Q_{\mu} g\right|\right)^{q}\right)^{1 / q}\right\|_{L^{p}(\mathbf{R})} .
$$

See $[7,8]$ for a detailed description of the Triebel-Lizorkin spaces which occur in Fourier analysis. The parameters $\alpha, q, p$ are assumed to satisfy $\alpha \in \mathbf{R}, 1<p<\infty$, and $1<q \leq \infty$, with the usual interpretation if $q=\infty$. The space $H_{p}^{\alpha q}$ is defined to be the completion of $L_{f}^{2}$ with respect to the $\|\cdot\|_{H_{p}^{\alpha q}}$ norm.

One of the main results in [1] is that the space $H_{p}^{\alpha q}$ is essentially independent of the particular choice of $\varphi$ chosen to satisfy conditions (i), (ii). To be precise, suppose $\varphi^{(1)}, \varphi^{(2)}$ are two different $C^{\infty}$ functions satisfying (i), (ii), and let $H_{p}^{\alpha q}(1), H_{p}^{\alpha q}(2)$ denote the corresponding spaces. Then Theorem 1.1 of [1] states that $H_{p}^{\alpha q}(1)$ and $H_{p}^{\alpha q}(2)$ are identical as sets and have equivalent norms. Theorem 1.2 of [1]

Received by the editors January 3, 1995

1991 Mathematics Subject Classification. Primary 42C10.

(C)1996 American Mathematical Society 
states that the spaces $H_{p}^{02}$ and $L^{p}$ are isomorphic and have equivalent norms, as is expected.

A function $m: \mathbf{R}_{+} \rightarrow \mathbf{C}$ will be called an $H_{p}^{\alpha q}$ Hermite multiplier if the operator $m(H): L_{f}^{2} \rightarrow L_{f}^{2}$ has a bounded linear extension to $H_{p}^{\alpha q}$.

Theorem 1. Let $\alpha \in \mathbf{R}, 1<p, q<\infty$. Suppose $m: \mathbf{R}_{+} \rightarrow \mathbf{C}$ is bounded and satisfies $\left|m^{\prime}(\kappa)\right| \leq c \kappa^{-1}$. Then $m$ is an $H_{p}^{\alpha q}$ Hermite multiplier.

Note that this is directly analogous to Mihlin's theorem [2] for Fourier multipliers. Thangavelu $[5,6]$ first proved this theorem for $L^{p}$ spaces (the $\alpha=0, q=2$ case) using special $g$-functions based on the Hermite semigroup. Section 2 of this paper contains a natural, alternative approach to the proof of Theorem 1. Of course the derivative condition on $m$ in Theorem 1 can be replaced by a difference condition. Let $\Delta m(2 k+1):=m(2(k+1)+1)-m(2 k+1)$. In the proof we only need $m$ to satisfy $|\Delta m(2 k+1)| \leq c(1+k)^{-1}$ for $k \in \mathbf{N}_{0}$, which is certainly implied by the condition given on $m^{\prime}$.

Next we consider pseudo-multipliers. Let $a: \mathbf{R} \times \mathbf{R}_{+} \rightarrow \mathbf{C}$ be bounded, and for $g \in L_{f}^{2}$ define

$$
A g(x)=\sum_{k=0}^{\infty} a(x, 2 k+1)\left\langle g, h_{k}\right\rangle h_{k}(x) .
$$

Theorem 2. Suppose $a(x, \kappa)$ is measurable in the $x$ variable for each fixed $\kappa$, and satisfies $\left|\partial_{\kappa}^{\gamma} a(x, \kappa)\right| \leq c(1+\kappa)^{-\gamma}$ for $0 \leq \gamma \leq 5$. If the operator $A$ is bounded on $L^{2}$, then $A$ also extends to a bounded operator on $L^{p}$ for $1<p<2$.

Using a method from [1] we establish uniform weak- $\left(L^{1}, L^{1}\right)$ bounds on certain truncated versions of $A$, from which Theorem 2 follows by Marcinkiewicz interpolation. See Section 3.

\section{Multipliers}

We begin by describing the main steps toward proving Theorem 1. As in [1], let $\psi: \mathbf{R} \rightarrow \mathbf{C}$ satisfy the same conditions (i), (ii) as $\varphi$, and the condition

$$
\sum_{\mu=0}^{\infty} \varphi\left(2^{-\mu} x\right) \psi\left(2^{-\mu} x\right)=1 \quad \text { for all } x \geq 1 .
$$

Let $\rho(x)=\varphi(2 x) \psi(2 x)+\varphi(x) \psi(x)+\varphi\left(2^{-1} x\right) \psi\left(2^{-1} x\right)$. For $\mu \in \mathbf{N}_{0}$ define $T_{\mu}=$ $\rho\left(2^{-\mu} H\right)$. Note that $Q_{\mu}=T_{\mu} Q_{\mu}$. Now let $m$ be as in Theorem 1 , and for each $\mu \in \mathbf{N}_{0}$ let $W_{\mu}=m(H) T_{\mu}$. Let $L^{2}\left(l^{2}\right)_{f}$ denote the subspace of $L^{2}\left(l^{2}\right)$ consisting of sequences $\left\{g_{\mu}\right\}_{\mu=0}^{\infty}$ such that only finitely many $g_{\mu}$ are nonvanishing. Finally, define

$$
W: L^{2}\left(l^{2}\right)_{f} \rightarrow L^{2}\left(l^{2}\right)_{f}
$$

by $W\left(\left\{g_{\mu}\right\}_{\mu=0}^{\infty}\right)=\left\{W_{\mu} g_{\mu}\right\}_{\mu=0}^{\infty}$. It is easy to see that for $g \in L_{f}^{2}$,

$$
\|m(H) g\|_{H_{p}^{\alpha q}}=\left\|W\left(\left\{2^{\mu \alpha} Q_{\mu} g\right\}\right)\right\|_{L^{p}\left(l^{q}\right)} .
$$

Therefore, to prove Theorem 1 it suffices to show that $W$ is bounded on $L^{2}\left(l^{2}\right)_{f} \cap$ $L^{p}\left(l^{q}\right)$ in the $L^{p}\left(l^{q}\right)$ norm topology.

Lemma 2.1. Let $1<q<\infty$. Then $W$ has a bounded linear extension to $L^{q}\left(l^{q}\right)$.

Lemma 2.2. Let $1<q<\infty$. Then $W$ is weak- $\left(L^{1}\left(l^{q}\right), L^{1}\left(l^{q}\right)\right)$ bounded. 
By Marcinkiewicz interpolation, these two lemmas suffice to show that $W$ is bounded on $L^{2}\left(l^{2}\right)_{f} \cap L^{p}\left(l^{q}\right)$ in the $L^{p}\left(l^{q}\right)$ norm topology, for $1<p \leq q<\infty$. The case $1<q \leq p<\infty$ follows from the facts that: (1) $L^{p^{\prime}}\left(l^{q^{\prime}}\right)$ is the dual of $L^{p}\left(l^{q}\right)$, and (2) Lemmas 2.1 and 2.2 continue to hold if $m$ and $\rho$ are replaced by their complex conjugates.

The proofs of Lemmas 2.1 and 2.2 depend on integral estimates for the kernels of the $W_{\mu}$ operators. First we need

Lemma 2.3. There exist constants $c_{1}, c_{2}>0$ independent of $L \geq 1$ such that

$$
\sum_{k=0}^{L} h_{k}^{2}(x) \leq c_{1} L^{1 / 2} e^{-c_{2} L^{-1} x^{2}}
$$

Proof. We recall the argument used to prove Lemma 3.2.1 in [6]. If $0<r<1$, then by Mehler's formula

$$
\sum_{k=0}^{L} h_{k}^{2}(x) \leq r^{-L} \sum_{k=0}^{\infty} r^{k} h_{k}^{2}(x)=\pi^{-1 / 2} r^{-L}\left(1-r^{2}\right)^{-1 / 2} e^{-\frac{1-r}{1+r} x^{2}}
$$

Substituting $r=e^{-1 / L}$ we get

$$
\sum_{k=0}^{L} h_{k}^{2}(x) \leq c_{1} L^{1 / 2} e^{-c_{2} L^{-1} x^{2}}
$$

Lemma 2.4. There exist constants $0<c_{1}, c_{2}<\infty$ such that for every $t>0$, $\mu \in \mathbf{N}_{0}$, and $y \in \mathbf{R}$,

$$
\int_{|x-y| \geq t}\left|W_{\mu}(x, y)\right| d x \leq c_{1}\left(2^{\mu / 2} t\right)^{-1 / 2} e^{-c_{2} 2^{-\mu} y^{2}}
$$

Proof. Inequality (2) follows from

$$
\int_{-\infty}^{\infty}(x-y)^{2}\left|W_{\mu}(x, y)\right|^{2} d x \leq c_{1} 2^{-\mu / 2} e^{-c_{2} 2^{-\mu} y^{2}}
$$

by an application of Schwarz's inequality. To prove (3) we use a simple case of Thangavelu's Lemma 3.2.3 ([6], p. 72):

$$
(x-y) W_{\mu}(x, y)=\frac{1}{2}(B-A) \Delta W_{\mu}(x, y) .
$$

Here $A=-\frac{\partial}{\partial x}+x, B=-\frac{\partial}{\partial y}+y$, and

$$
\Delta W_{\mu}(x, y):=\sum_{k=0}^{\infty} \Delta\left(m(2 k+1) \rho\left(2^{-\mu}(2 k+1)\right)\right) h_{k}(x) h_{k}(y) .
$$

Identity (4) is easily derived from the recursion relation

$$
2 x h_{k}(x)=(2 k+2)^{1 / 2} h_{k+1}(x)+(2 k)^{1 / 2} h_{k-1}(x),
$$

together with the fact that

$$
\left(-\frac{d}{d x}+x\right) h_{k}(x)=(2 k+2)^{1 / 2} h_{k+1}(x) .
$$


Substituting (4) in (3), we get

(5)

$$
\begin{aligned}
\int_{-\infty}^{\infty}( & (x-y)^{2}\left|W_{\mu}(x, y)\right|^{2} d x \\
\leq & c \int_{-\infty}^{\infty}\left|B \Delta W_{\mu}(x, y)\right|^{2} d x+c \int_{-\infty}^{\infty}\left|A \Delta W_{\mu}(x, y)\right|^{2} d x \\
\leq & c \sum_{k=0}^{\infty}\left|\Delta m(2 k+1) \rho\left(2^{-\mu}(2 k+1)\right)+m(2 k+3) \Delta \rho\left(2^{-\mu}(2 k+1)\right)\right|^{2} \\
& \cdot(2 k+2)\left(h_{k+1}^{2}(y)+h_{k}^{2}(y)\right) \\
\leq & c \sum_{k=0}^{\infty}\left(\left|(1+k)^{-1} \rho\left(2^{-\mu}(2 k+1)\right)\right|+\left|m(2 k+3) 2^{-\mu} \rho^{\prime}\left(2^{-\mu} \xi(k)\right)\right|\right)^{2} \\
& \cdot(2 k+2)\left(h_{k+1}^{2}(y)+h_{k}^{2}(y)\right),
\end{aligned}
$$

where each $\xi(k)$ is between $2 k+1$ and $2 k+3$. Since $\rho$ is compactly supported away from the origin, there exist integers $0<N_{1}<N_{2}$ independent of $\mu \in \mathbf{N}_{0}$ such that the terms in (5) vanish unless $2^{\mu} N_{1} \leq k \leq 2^{\mu} N_{2}$. Thus (5) is bounded by

$$
c \sum_{k=2^{\mu} N_{1}}^{2^{\mu} N_{2}+1} 2^{-2 \mu} 2^{\mu} h_{k}^{2}(y) \leq c_{1} 2^{-\mu / 2} e^{-c_{2} 2^{-\mu} y^{2}}
$$

by an application of Lemma 2.3 .

Lemma 2.5. There exists a constant $c<\infty$ such that for every $t>0, \mu \in \mathbf{N}_{0}$, and $y, z \in \mathbf{R}$ with $|y-z| \leq t$,

(6)

$$
\int_{|x-z| \geq 2 t}\left|W_{\mu}(x, y)-\left(W_{\mu}(x, z)+(y-z) D_{2} W_{\mu}(x, z)\right)\right| d x \leq c\left(2^{\mu / 2} t\right)^{3 / 2} .
$$

Proof. Let $J$ denote the interval with endpoints $y, z$. We can rewrite the left side of (6) as

$$
\begin{aligned}
\int_{|x-z| \geq 2 t} \mid & \int_{J}(y-u) D_{2}^{2} W_{\mu}(x, u) d u \mid d x \\
& \leq \int_{J}|y-u| \int_{|x-z| \geq 2 t}\left|D_{2}^{2} W_{\mu}(x, u)\right| d x d u \\
& \leq t^{2} \sup _{u \in J} \int_{|x-z| \geq 2 t}\left|D_{2}^{2} W_{\mu}(x, u)\right| d x .
\end{aligned}
$$

We estimate the last integral using

$$
D_{2}^{2} W_{\mu}(x, u)=\left(D_{2}^{2}-u^{2}\right) W_{\mu}(x, u)+u^{2} W_{\mu}(x, u)=I+I I .
$$

Note that

$$
I=-2^{\mu} \sum_{k=0}^{\infty} m(2 k+1) \rho\left(2^{-\mu}(2 k+1)\right)\left(2^{-\mu}(2 k+1)\right) h_{k}(x) h_{k}(y) .
$$

Hence, by the method of proving Lemma 2.4 we get

$$
t^{2} \sup _{u \in J} \int_{|x-z| \geq 2 t}|I| d x \leq t^{2} \sup _{u \in J} \int_{|x-u| \geq t}|I| d x \leq c t^{2} 2^{\mu}\left(2^{\mu / 2} t\right)^{-1 / 2} .
$$


Again using Lemma 2.4, we have

$$
t^{2} \sup _{u \in J} \int_{|x-z| \geq 2 t}|I I| d x \leq c\left(2^{\mu / 2} t\right)^{2} \sup _{u \in J} 2^{-\mu} u^{2}\left(2^{\mu / 2} t\right)^{-1 / 2} e^{-c_{2} 2^{-\mu} u^{2}} \leq c\left(2^{\mu / 2} t\right)^{3 / 2} .
$$

Proof of Lemma 2.1. It suffices to show that the operators $W_{\mu}, \mu \in \mathbf{N}_{0}$, are uniformly bounded on $L^{q}$. This is trivial for $q=2$, so it suffices (by interpolation and duality) to show that the $W_{\mu}$ operators are uniformly weak- $\left(L^{1}, L^{1}\right)$ bounded. To do this we must show that there exists a constant $c<\infty$ independent of $f \in L^{1}$, $\lambda>0$, and $\mu \in \mathbf{N}_{0}$ such that

$$
\left|\left\{x:\left|W_{\mu} f(x)\right|>\lambda\right\}\right| \leq \frac{c}{\lambda}\|f\|_{L^{1}} .
$$

This will be a routine application of Lemmas 2.4 and 2.5. So fix $f \in L^{1}$ and $\lambda>0$, and apply the Calderón-Zygmund lemma to get a collection of disjoint dyadic open intervals $\left\{I_{j}\right\}$ such that

(a) $|f(x)| \leq \lambda$ for a.e. $x \in \mathbf{R} \backslash \cup_{j} I_{j}$,

(b) $\sum_{j}\left|I_{j}\right| \leq \frac{1}{\lambda}\|f\|_{L^{1}}$,

(c) $\lambda \leq \frac{1}{\left|I_{j}\right|} \int_{I_{j}}|f(x)| d x \leq 2 \lambda$ for all $j$.

Let $z_{j}$ denote the centerpoint of $I_{j}$, and for $x \in I_{j}$ let

$$
g(x)=\frac{1}{\left|I_{j}\right|} \int_{I_{j}} f(y) d y+\frac{12\left(x-z_{j}\right)}{\left|I_{j}\right|^{3}} \int_{I_{j}} f(y)\left(y-z_{j}\right) d y .
$$

Also, if $x \in I_{j}$, let $b(x)=f(x)-g(x)$. For $x \notin \bigcup_{j} I_{j}$, let $g(x)=f(x)$ and $b(x)=0$. Thus $f=g+b$ everywhere. Note that if $x \in I_{j}$, then $|g(x)| \leq 8 \lambda$. Also, for a.e. $x \notin \bigcup_{j} I_{j},|g(x)| \leq \lambda$.

By the standard argument $\|g\|_{L^{2}}^{2} \leq c \lambda\|f\|_{L^{1}}$. So, by Chebyshev's inequality

$$
\left|\left\{x:\left|W_{\mu} g(x)\right|>\lambda / 2\right\}\right| \leq \frac{4}{\lambda^{2}}\left\|W_{\mu} g\right\|_{L^{2}}^{2} \leq \frac{c}{\lambda}\|f\|_{L^{1}} .
$$

Next we have to prove the correct sort of estimate for $\left|\left\{x:\left|W_{\mu} b(x)\right|>\lambda / 2\right\}\right|$. Define $I_{j}^{*}=\left(z_{j}-\left|I_{j}\right|, z_{j}+\left|I_{j}\right|\right)$. Since $\left|\bigcup_{j} I_{j}^{*}\right| \leq \frac{2}{\lambda}\|f\|_{L^{1}}$, it suffices to estimate

$$
\left|\left\{x \in \mathbf{R} \backslash \bigcup_{j} I_{j}^{*}:\left|W_{\mu} b(x)\right|>\lambda / 2\right\}\right| .
$$

For each $j$ let $b_{j}=b \cdot \chi_{I_{j}}$. Then $b=\sum_{j} b_{j}$ a.e., $\int b_{j}(x)\left(x-z_{j}\right) d x=0$, and $\int b_{j}(x) d x=0$. By Chebyshev's inequality

$$
\left|\left\{x \in \mathbf{R} \backslash \bigcup_{j} I_{j}^{*}:\left|W_{\mu} b(x)\right|>\lambda / 2\right\}\right| \leq \frac{2}{\lambda} \sum_{j} \int_{\mathbf{R} \backslash I_{j}^{*}}\left|W_{\mu} b_{j}(x)\right| d x .
$$

For each $j$ define the kernel

$$
L_{\mu}^{j}(x, y)= \begin{cases}W_{\mu}(x, y) & \text { if } 2^{\mu / 2}\left|I_{j}\right| \geq 1 \\ W_{\mu}(x, y)-\left(W_{\mu}\left(x, z_{j}\right)+\left(y-z_{j}\right) D_{2} W_{\mu}\left(x, z_{j}\right)\right) & \text { if } 2^{\mu / 2}\left|I_{j}\right|<1\end{cases}
$$

Because of the vanishing moment conditions imposed on $b_{j}$, we have

$$
\begin{aligned}
\int_{\mathbf{R} \backslash I_{j}^{*}}\left|W_{\mu} b_{j}(x)\right| d x & =\int_{\mathbf{R} \backslash I_{j}^{*}}\left|\int_{I_{j}} L_{\mu}^{j}(x, y) b_{j}(y) d y\right| d x \\
& \leq \int_{I_{j}}\left|b_{j}(y)\right| \int_{\mathbf{R} \backslash I_{j}^{*}}\left|L_{\mu}^{j}(x, y)\right| d x d y
\end{aligned}
$$


Now according to Lemmas 2.4 and 2.5 we see that (7) is bounded by

$$
\frac{c}{\lambda} \sum_{j} \min \left\{\left(2^{\mu / 2}\left|I_{j}\right|\right)^{-1 / 2},\left(2^{\mu / 2}\left|I_{j}\right|\right)^{3 / 2}\right\} \int_{I_{j}}\left|b_{j}(y)\right| d y \leq \frac{c}{\lambda}\|f\|_{L^{1}} .
$$

Proof of Lemma 2.2. We need to show that there exists a constant $c<\infty$ independent of $\left\{f_{\mu}\right\} \in L^{1}\left(l^{q}\right), \lambda>0$ such that

$$
\left|\left\{x:\left(\sum_{\mu=0}^{\infty}\left|W_{\mu} f_{\mu}(x)\right|^{q}\right)^{1 / q}>\lambda\right\}\right| \leq \frac{c}{\lambda}\left\|\left\{f_{\mu}\right\}\right\|_{L^{1}\left(l^{q}\right)} .
$$

So fix $\left\{f_{\mu}\right\} \in L^{1}\left(l^{q}\right)$ and $\lambda>0$, let $h(x)=\left(\sum_{\mu=0}^{\infty}\left|f_{\mu}(x)\right|^{q}\right)^{1 / q}$, and apply the Calderón-Zygmund lemma to get a collection of disjoint open intervals $\left\{I_{j}\right\}$ such that

(a) $|h(x)| \leq \lambda$ for a.e. $x \in \mathbf{R} \backslash \bigcup_{j} I_{j}$,

(b) $\quad \sum_{j}\left|I_{j}\right| \leq \frac{1}{\lambda}\|h\|_{L^{1}}$

(c) $\quad \lambda \leq \frac{1}{\left|I_{j}\right|} \int_{I_{j}}|h(x)| d x \leq 2 \lambda$ for all $j$.

Again let $z_{j}$ denote the centerpoint of $I_{j}$, and for $x \in I_{j}$ let

$$
g_{\mu}(x)=\frac{1}{\left|I_{j}\right|} \int_{I_{j}} f_{\mu}(y) d y+\frac{12\left(x-z_{j}\right)}{\left|I_{j}\right|^{3}} \int_{I_{j}} f_{\mu}(y)\left(y-z_{j}\right) d y .
$$

For $x \in I_{j}$ let $b_{\mu}(x)=f_{\mu}(x)-g_{\mu}(x)$, and for $x \notin \bigcup_{j} I_{j}$ let $g_{\mu}(x)=f_{\mu}(x), b_{\mu}(x)=0$. If $x \in I_{j}$ we have by Minkowski's inequality

$$
\begin{aligned}
& \left(\sum_{\mu=0}^{\infty}\left|g_{\mu}(x)\right|^{q}\right)^{1 / q} \\
& \quad \leq\left(\sum_{\mu=0}^{\infty}\left|\frac{1}{\left|I_{j}\right|} \int_{I_{j}} f_{\mu}(y) d y\right|^{q}\right)^{1 / q}+\left(\sum_{\mu=0}^{\infty}\left|\frac{12\left(x-z_{j}\right)}{\left|I_{j}\right|^{3}} \int_{I_{j}} f_{\mu}(y)\left(y-z_{j}\right) d y\right|^{q}\right)^{1 / q} \\
& \quad \leq \frac{1}{\left|I_{j}\right|} \int_{I_{j}}\left(\sum_{\mu=0}^{\infty}\left|f_{\mu}(y)\right|^{q}\right)^{1 / q} d y+\frac{3}{\left|I_{j}\right|} \int_{I_{j}}\left(\sum_{\mu=0}^{\infty}\left|f_{\mu}(y)\right|^{q}\right)^{1 / q} d y \\
& \quad \leq 8 \lambda .
\end{aligned}
$$

It follows that $\left\|\left\{g_{\mu}\right\}\right\|_{L^{q}\left(l^{q}\right)}^{q} \leq c \lambda^{q-1}\left\|\left\{f_{\mu}\right\}\right\|_{L^{1}\left(l^{q}\right)}$, and therefore by Chebyshev's inequality and Lemma 2.1,

$$
\left|\left\{x:\left(\sum_{\mu=0}^{\infty}\left|W_{\mu} g_{\mu}(x)\right|^{q}\right)^{1 / q}>\lambda / 2\right\}\right| \leq \frac{2^{q}}{\lambda^{q}}\left\|\left\{W_{\mu} g_{\mu}\right\}\right\|_{L^{q}\left(l^{q}\right)}^{q} \leq \frac{c}{\lambda}\left\|\left\{f_{\mu}\right\}\right\|_{L^{1}\left(l^{q}\right)} .
$$

Next we have to estimate $\left|\left\{x:\left(\sum_{\mu=0}^{\infty}\left|W_{\mu} b_{\mu}(x)\right|^{q}\right)^{1 / q}>\lambda / 2\right\}\right|$. As in the proof of Lemma 2.1 it suffices to handle

$$
\begin{aligned}
\mid\{x \in & \left.\mathbf{R} \backslash \bigcup_{j} I_{j}^{*}:\left(\sum_{\mu=0}^{\infty}\left|W_{\mu} b_{\mu}(x)\right|^{q}\right)^{1 / q}>\lambda / 2\right\} \mid \\
& \leq \frac{2}{\lambda} \sum_{j} \int_{\mathbf{R} \backslash I_{j}^{*}}\left(\sum_{\mu=0}^{\infty}\left|W_{\mu} b_{\mu, j}(x)\right|^{q}\right)^{1 / q} d x .
\end{aligned}
$$


Here of course $b_{\mu, j}=b_{\mu} \cdot \chi_{I_{j}}$. Let $L_{\mu}^{j}$ be as in the proof of Lemma 2.1. Then by Minkowski's inequality and lemmas Lemmas 2.4 and 2.5

$$
\begin{aligned}
\int_{\mathbf{R} \backslash I_{j}^{*}} & \left(\sum_{\mu=0}^{\infty}\left|W_{\mu} b_{\mu, j}(x)\right|^{q}\right)^{1 / q} d x \\
& =\int_{\mathbf{R} \backslash I_{j}^{*}}\left(\sum_{\mu=0}^{\infty}\left|\int_{I_{j}} L_{\mu}^{j}(x, y) b_{\mu, j}(y) d y\right|^{q}\right)^{1 / q} d x \\
& \leq \int_{\mathbf{R} \backslash I_{j}^{*}} \int_{I_{j}}\left(\sum_{\mu=0}^{\infty}\left|L_{\mu}^{j}(x, y) b_{\mu, j}(y)\right|^{q}\right)^{1 / q} d y d x \\
& \leq \int_{I_{j}}\left(\sum_{\mu=0}^{\infty}\left|b_{\mu, j}(y)\right|^{q}\right)^{1 / q}\left(\int_{\mathbf{R} \backslash I_{j}^{*}} \sum_{\mu=0}^{\infty}\left|L_{\mu}^{j}(x, y)\right| d x\right) d y \\
& \leq c \int_{I_{j}}\left(\sum_{\mu=0}^{\infty}\left|b_{\mu, j}(y)\right|^{q}\right)^{1 / q}\left(\sum_{\mu=0}^{\infty} \min \left\{\left(2^{\mu / 2}\left|I_{j}\right|\right)^{-1 / 2},\left(2^{\mu / 2}\left|I_{j}\right|\right)^{3 / 2}\right\}\right) d y \\
& \leq c \int_{I_{j}}\left(\sum_{\mu=0}^{\infty}\left|b_{\mu, j}(y)\right|^{q}\right)^{1 / q} d y \\
& \leq c \int_{I_{j}}\left(\sum_{\mu=0}^{\infty}\left|f_{\mu}(y)\right|^{q}\right)^{1 / q} d y
\end{aligned}
$$

with $c$ independent of $\left|I_{j}\right|$. Substituting this in (8) finishes the proof.

\section{Pseudo-multipliers}

In this section we prove Theorem 2. Let $P_{\mu}=\pi\left(2^{-\mu} H\right)$, where $\pi(x)$ is a $C^{\infty}$ function supported on $\left[\frac{1}{2}, 2\right]$ with the property that $\sum_{\mu=0}^{\infty} \pi\left(2^{-\mu} x\right)=1$ for all $x \geq 1$.

Lemma 3.1. Let $a(x, \kappa)$ be as in the statement of Theorem 2, and suppose that the associated operator $A$ is bounded on $L^{2}$. Then the operators $\sum_{\mu=0}^{N} A P_{\mu}$ are weak- $\left(L^{1}, L^{1}\right)$ bounded, uniformly in $N$.

Proof. We begin by recalling how to estimate the kernel $K_{\mu}(x, y)$ of the operator $A P_{\mu}$. Let $a_{\mu}(x, \kappa)=a(x, \kappa) \pi\left(2^{-\mu} \kappa\right)$, and let $\hat{a}_{\mu}(x, \xi)$ denote the Fourier transform of $a_{\mu}$ in its second variable. As in the derivation of (7) in $\S 3$ of [1], we have the representation

$$
K_{\mu}(x, y)=c \int_{-\infty}^{\infty} \hat{a}_{\mu}(x, \xi / 2) e^{i \xi / 2}\left(1-e^{i 2 \xi}\right)^{-1 / 2} e^{-\frac{i}{2}\left(\left(x^{2}+y^{2}\right) \cot \xi-2 x y \csc \xi\right)} d \xi,
$$

where $c$ is some unimportant constant. Now the conditions on $a$ imply that for every $l \in \mathbf{N}_{0}$ there exists a constant $c_{l}$ independent of $\mu \in \mathbf{N}_{0}$ such that $\left|\partial_{\xi}^{l} \hat{a}_{\mu}(x, \xi)\right| \leq$ $c_{l} 2^{\mu(1+l)}\left(1+2^{\mu}|\xi|\right)^{-5}$. It follows by inspection of the proof of Lemma 1.1 in [1] that there exists a constant $c$ independent of $\mu \in \mathbf{N}_{0}$ such that

$$
\left|K_{\mu}(x, y)\right| \leq \sum_{\sigma= \pm 1} \frac{c 2^{\mu / 2}}{\left(1+2^{\mu / 2}|x+\sigma y|\right)^{4}}
$$

and

$$
\left|\partial_{y}^{2} K_{\mu}(x, y)\right| \leq \sum_{\sigma= \pm 1} \frac{c 2^{3 \mu / 2}}{\left(1+2^{\mu / 2}|x+\sigma y|\right)^{2}}
$$


The proof is finished with a simple modification of the proof of Lemma 2.1, which we very briefly indicate. Fix $f \in L^{1}$ and $\lambda>0$, and let $\left\{I_{j}\right\}, g(x), b(x)$, etc., be as in the proof of Lemma 2.1. From the $L^{2}$-boundedness of $A$ and the uniform $L^{2}$-boundedness of the operators $\sum_{\mu=0}^{N} P_{\mu}$ we get

$$
\left|\left\{x:\left|\sum_{\mu=0}^{N} A P_{\mu} g(x)\right|>\lambda / 2\right\}\right| \leq \frac{c}{\lambda}\|f\|_{L^{1}} .
$$

Now let $L_{\mu}^{j}(x, y)$ be defined as in the proof of Lemma 2.1, except with $K_{\mu}(x, y)$ in place of $W_{\mu}(x, y)$. Then from (9) and (10) we get

$$
\begin{aligned}
\int_{\mathbf{R} \backslash I_{j}^{*}}\left|\sum_{\mu=0}^{N} A P_{\mu} b_{j}(x)\right| d x & \leq \int_{I_{j}}\left|b_{j}(y)\right|\left(\int_{\mathbf{R} \backslash I_{j}^{*}} \sum_{\mu=0}^{N}\left|L_{\mu}^{j}(x, y)\right| d x\right) d y \\
& \leq c \int_{I_{j}}\left|b_{j}(y)\right|\left(\sum_{\mu=0}^{\infty} \min \left\{\left(2^{\mu / 2}\left|I_{j}\right|\right)^{-3}, 2^{\mu / 2}\left|I_{j}\right|\right\}\right) d y \\
& \leq c \int_{I_{j}}\left|b_{j}(y)\right| d y .
\end{aligned}
$$

Proof of Theorem 2. By Lemma 3.1 and the Marcinkiewicz interpolation theorem, each of the operators $\sum_{\mu=0}^{N} A P_{\mu}$ has a bounded linear extension to $L^{p}$, for $1<$ $p \leq 2$. Moreover, the operator norms $\left\|\sum_{\mu=0}^{N} A P_{\mu}\right\|_{L^{p} \rightarrow L^{p}}$ are bounded uniformly in $N$. Now let $g \in L_{f}^{2}$. Then $A g=\sum_{\mu=0}^{N} A P_{\mu} g$ for a large enough choice of $N$. Hence $\|A g\|_{L^{p}} \leq c\|g\|_{L^{p}}$. The proof is finished by recalling that $L_{f}^{2}$ is dense in $L^{p}$ (see for example [3], Lemma 2). It would be interesting to find natural criteria for the $L^{2}$-boundedness of a Hermite pseudo-multiplier, since the standard methods for obtaining $L^{2}$-boundedness of an ordinary pseudo-differential operator (as in [4]) do not seem to be applicable here.

\section{REFERENCES}

1. J. Epperson, Triebel-Lizorkin spaces for Hermite expansions, Studia Math. 114 (1995), 87103.

2. S.G. Mihlin, On the multipliers of Fourier integrals, Dokl. Acad. Nauk SSSR N.S. 109 (1956), 701-703 (Russian). MR 18:304a

3. B. Muckenhoupt, Mean convergence of Hermite and Laguerre series II, Trans. Amer. Math. Soc. 147 (1970), 433-460. MR 41:711

4. E.M. Stein, Harmonic Analysis, Princeton University Press, 1993. MR 95c:42002

5. S. Thangavelu, Multipliers for Hermite expansions, Revist. Mat. Ibero. 3 (1987), 1-24. MR 90h: 42043

6. S. Thangavelu, Lectures on Hermite and Laguerre Expansions, Mathematical Notes 42, Princeton University Press, 1993. MR 94i:42001

7. H. Triebel, Theory of Function Spaces, Birkhäuser Verlag, Basel (Monographs in Mathematics, Vol. 78), 1983. MR 86j:46026

8. H. Triebel, Theory of Function Spaces II, Birkhäuser Verlag, Basel (Monographs in Mathematics, Vol. 84), 1992. MR 93f:46029

Department of Mathematics and Statistics, University of New Mexico, Albuquerque, New Mexico 87131

E-mail address: jeppers@math.unm.edu 